\title{
Research on the Denoising of the Beidou Carrier Signal based on EEMD Algorithm with Adaptive Reconstruction Optimizing of IMF Numbers
}

\author{
WEI LI ${ }^{1}$, KAI ZHANG ${ }^{2 *}$, GANG LV ${ }^{1}$, GUIBAO XU ${ }^{3}$, ANYI XU ${ }^{4}$ \\ 1. Advanced Manufacturing Engineering College of Hefei University, Hefei, 230601, CHINA \\ 2. National Synchrotron Radiation Laboratory, University of Science and Technology of China, Hefei, \\ 230029, CHINA \\ 3. Anhui Beidou Satellite Navigation Platform Co. Ltd. Hefei, 230011, CHINA \\ 4. Hefei Danning Project Management\&Consulting Co. Ltd. Hefei, 230031, CHINA
}

\begin{abstract}
The Beidou carrier signal is coupled into a certain noise during propagation and reception, and these noise will directly affect the processing procedure associated with it. To deal with the problem of the influence due to the manually setting the IMF (Intrinsic Mode Function) component number for the reconstruction signal, a new measuring index that used for finding the optimal IMF components to reconstruct the signal has been designed in this paper. The index has taken the shape of the signal, signal noise ratio and correlation index into consideration. Upon on the basis, an adaptive index optimization Ensemble Empirical Mode Decomposition (AIO-EEMD) algorithm has been proposed in this paper. To verify the validity of the algorithm, four different algorithms are used to denoised the collected Beidou signal. The experiment results show that the noise reduction using the AIO-EEMD method can not only automatically obtain the optimal IMF components number, but also has a significant advantage over the other three methods.
\end{abstract}

Key words: Beidou carrier signal, adaptive, reconstructed component number, EEMD, noise reduction Received: April 11, 2020. Revised: October 29, 2020. Accepted: November 17, 2020. Published: November 30 , 2020.

\section{Introduction}

The self-developed Beidou satellite navigation system of China is one of the three mature satellite navigation systems in the world. The continual evolution of the Beidou system has a great significance for the economic development and national defense construction. The Beidou carrier signal received by the positioning device is susceptible to be affected by the noise during the long distance transmission and reception. It will cause errors in the carrier signal, which will lead to the decrease of the positioning accuracy. Thus, how to reduce or even eliminate the noise coupled into the Beidou satellite signal has become a vital problem which need to be solved [1].

The wavelet decomposition has been commonly used in the denoising processing of 
various signals [2]. N. E. Huang, etc. has proposed a modern signal processing method: Ensemble Empirical Mode Decomposition (EEMD) at the end of 20th century, and it has been widely used in the extraction of useful signals under complex noise background [3]. Combining the characteristics of Beidou carrier signal and the application scenarios of the above methods, it can be deduced that the EEMD method is a suitable method to process the Beidou signal. As the key indicator in the reconstruction process, the number of IMF components in common EEMD algorithm is usually set manually based on experience, which will affect the accuracy of noise reduction $[4,5]$. Therefore, how to set the number of IMF components has become a problem that needs to be solved.

In order to deal with the problem mentioned before, a novel algorithm named adaptive index optimization EEMD (AIO-EEMD) is proposed in this paper. In this algorithm a new measuring index for finding the optimal IMF components has been designed to reconstruct the signal. Then, the proposed algorithm has been used on the carrier signal collected by a Beidou Locator and compared with other three methods. The results shows that the performance of this algorithm is superior to other methods, which indicates that the proposed algorithm is effective and validity.

\section{Definition of Beidou Satellite Signal}

The Beidou satellite signal is a kind of orthogonal modulation signal. The two components of the orthogonal signals are the navigation message and the ranging code which comes from I branch and Q branch [6]. Among them, I is an open branch which serves for ordinary civilian users, and $\mathrm{Q}$ is an authorized branch that only access to military and licensed users. Therefore, we only discuss the issue of the relevant noise reduction on branch I of the Beidou B1 signal (B1I) in this paper. The specific structure of the signal is shown in Fig.1 [7].

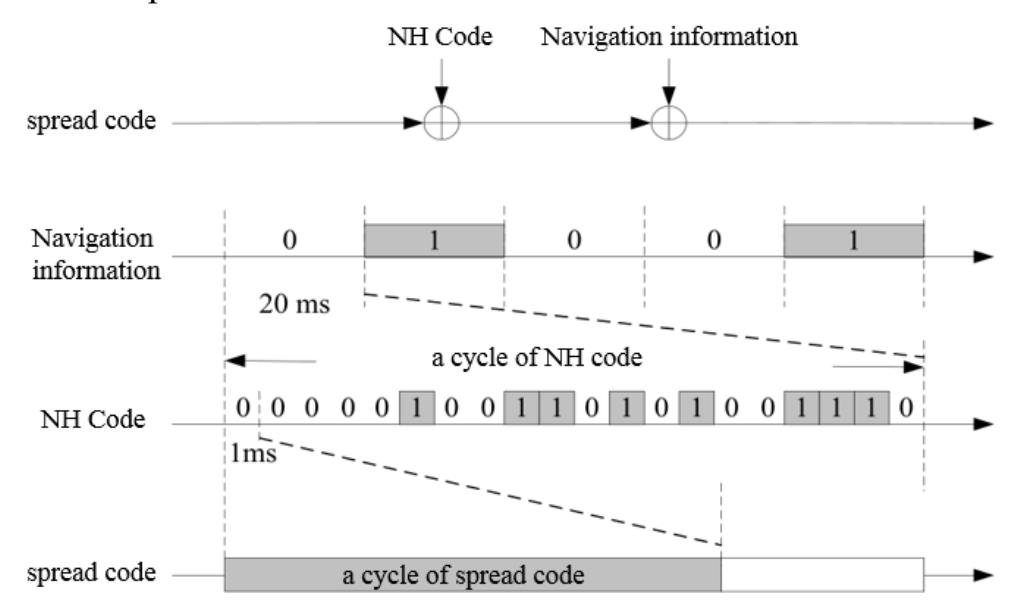

Fig.1 The structure of Beidou satellite navigation B1I signal

The B1I signal is composed of ranging code and navigation message which are modulated on the carrier signal. The frequency of the carrier signal transmitted by the same satellite is generated by a common clock source. The nominal frequency of B1I carrier signal is $1561.098 \mathrm{MHz}$ and the modulation mode is binary phase shift keying (BPSK). The transmitting mode of the B1I signal is the right-handed circular polarization (RHCP). The minimum power of the signal from the B1I branch by Beidou satellite to the output of the receiver antenna is $-163 \mathrm{dBW}$. It adopts the Code division multiple access (CDMA) to signal multiplexing mode. The bandwidth of the B1I is $4.092 \mathrm{MHz}$, and it's centered on the B1I signal carrier frequency. The ranging code rate is 2.046 Mcps and the 
signal's length is 2046. The expression of B1I carrier signal is as follows $[8,9]$.

$$
S^{j}(t)=A^{*} C^{j}(t)^{*} D^{j}(t) \cos \left(2 \pi f_{0} t+\varphi^{j}\right)
$$

Where, A is the amplitude of the B1I signal, C and $\mathrm{D}$ represent the ranging code and navigation message, respectively. The superscript letter $j$ represents the satellite number. $f_{0}$ is the carrier frequency and $\varphi$ represents the initial phase of the B1I carrier signal.

\section{EEMD algorithm and its key}

\section{indicators}

Before the EEMD algorithm was invented, the Empirical Mode Decomposition (EMD) algorithm was first proposed by Huang that used for the processing of nonlinear signal. Different from other methods that used for the analyzing of the non-stationary signal, the goal of EMD is to decompose the signal into instantaneous frequency with physical significance and then evaluate it [10]. EMD method is an empirical method that uses spline curve and its median value to decompose the original signal for several times. The density of the time serials points and amplitude in time domain are both reduced by half after each decomposition [11]. That is to say, the EMD method can realize the target of multi-scale decomposing in both the time domain and frequency domain. A finite number of Intrinsic Mode Functions (IMF) is obtained after decomposing process [12]. However, the mode mixing problem will be accompanied during the whole process. In other words, the useful signal and noise are simultaneously decomposed into the same order IMF, which may lead to the loss of the useful signals or the omission of the noise [13].

To solve the problem mentioned above, N. E. Huang etc. have proposed the Ensemble Empirical Mode Decomposition (EEMD) method. To obtain a sufficient number of extreme points that satisfy the smooth abnormal events processing demands, the first step in EEMD method is to superimpose Gaussian white noise into the original signal. After that, it's needed to get the mean value of IMF to overcome the problem of mode mixing [14, 15]. Since the superimposed Gaussian white noise has a characteristic of uncorrected zero-mean random, it will be removed from the final signal after an overall average. The steps of the EEMD method are as follows.

i. First, the Gauss white noises with unequal amplitude is superimposed on the signal to be processed. Next, we should adopt the EMD method to decompose the mixed signal for $\mathrm{k}$ times to get the corresponded IMF component $\mathrm{IMF}_{\mathrm{ik}}$ and the reminder component $\mathrm{R}_{\mathrm{ik}}$. The amplitude of the Gauss white noises should conform to the following formula.

$$
a=e k \text { or } \ln e+0.5 a \ln k=0
$$

Where, a is the amplitude of the white noise, e is the standard deviation of the original signal. It stands for the deviation between the original signal and the reconstruction signal with EMD method. Huang, etc. also pointed out that the amplitude of the white noise does not seriously affect the decomposition result unless it is infinitesimal or extreme large [16]. Further, the decomposition result of EEMD method is not going to change much because of the change in one or several parameters. In other words, this algorithm is adaptive.

ii. Then, calculate the overall average value of all the IMF components and the reminder component obtained with the EEMD method. The detailed expressions are as follows.

$$
I M F_{i}=\frac{1}{n} \sum_{k=1}^{n} I M F_{i k}
$$

Where, $\mathrm{n}$ is the number of EMD decomposition. The frequency of each IMF is 
different in EEMD process. The first to be decomposed is always with a high-frequency IMF, and the frequency is decrease in an exponential power of two. So the decomposition process can be considered as the results of multiple band-pass filtering, and the signal noise reduction can be achieved by constructing a low-pass filter [17]. The formula for signal reconstruction is as follows.

$$
x=\sum_{i=I}^{m} I M F_{i}
$$

Where, $\mathrm{i}$ is the components number of IMF, and it ranges from 1 to $\mathrm{m}$. It can be seen from the equation that the number of IMF components plays a vital role in constructing the EMD or EEMD low-pass filter. It not only represents the IMF component of the noise, but also stands for the useful signals' IMF. The value of I directly determines the accuracy of the EEMD noise reduction, and it usually adopts the posterior noise reduction performance index method to determinate its final values. The effect of noise reduction can be measured in terms of signal deformation and SNR. If the value of I is not unreasonable, it will cause the increase of the noise component or the loss of the useful signals's IMF components in low-pass filter, which will seriously affect the accuracy of the EEMD noise reduction $[18,19]$.

To solve the above problem, a new optimizing index of IMF numbers for the signal reconstruction in EEMD method has been designed in this paper. With this index, the EEMD algorithm can achieve adaptive operation and get the optimal reconstructed component number I. in this way, the influence of artificial setting on the noise reduction accuracy can be avoid. In this way, the influence of artificial setting I on noise reduction accuracy can be avoided.

\section{Adaptive EEMD algorithm with}

Index Optimizing of IMF Number for

\section{the Reconstruction (AIO-EEMD)}

\subsection{The design of Optimizing Index of IMF Number for the Reconstruction}

The design of optimizing index of IMF number for the reconstruction is as follows. The global best value in our algorithm is used to determinate whether the IMF component number I is optical. It can realize the noise reduction target without any manual assistance during the whole process. A new comprehensive indicator $r$ has been used to measure the noise reduction effect of the proposed EEMD algorithm. The indicator is made up of three parts, namely Signal to Noise Ratio (SNR), Standard Error (SE) and Correlation Coefficient (R). The formula of the four indicators are expressed as follows respectively.

$$
\begin{gathered}
r=\left|S N R+\frac{1}{S E}+R\right| \\
S N R=10 \log \frac{S}{N}=10 \log \left(\frac{\sum_{j=1}^{n} x^{\prime 2}(j)}{\sum_{j=1}^{n}\left[x(j)-x^{\prime}(j)\right]^{2}}\right) \\
S E=\sqrt{\frac{1}{n} \sum_{j=1}^{n}\left[x(j)-x^{\prime}(j)\right]^{2}} \\
R=\frac{\sum_{j=1}^{n}\left(x(j)-\overline{x_{i}}\right)\left(x^{\prime}(j)-\overline{x^{\prime}}\right)}{\sqrt{\sum_{j=1}^{n}(x(j)-\bar{x})^{2}} \sqrt{\sum_{j=1}^{n}\left(x^{\prime}(j)-\overline{x^{\prime}}\right)^{2}}}
\end{gathered}
$$

Where, $\mathrm{x}$ represents the original signal before denoising and $x$ ' represents the reconstructed signal after denoising with the current IMF number.

$$
\begin{gathered}
\bar{x}=\frac{1}{n} \sum_{j=1}^{n} x(j) \\
x^{\prime-}=\frac{1}{n} \sum_{j=1}^{n} x^{\prime}(j)
\end{gathered}
$$


The normalized denoising index $r$ synthesizes the information of SNR, shape details and approximation to the original signal of the Beidou carrier signal to measure the denoising effect. The larger is $r$, the better is the denoising effect.

\subsection{The design of the AIO-EEMD algorithm}

Combined the noise reduction index $r$ mentioned before and the EEMD algorithm, a new adaptive EEMD algorithm with Index Optimizing of IMF Number for the Reconstruction (AIO-EEMD) used in the noise reduction of Beidou carrier signal has been designed. The detailed calculation flowchart is shown in Fig.2.

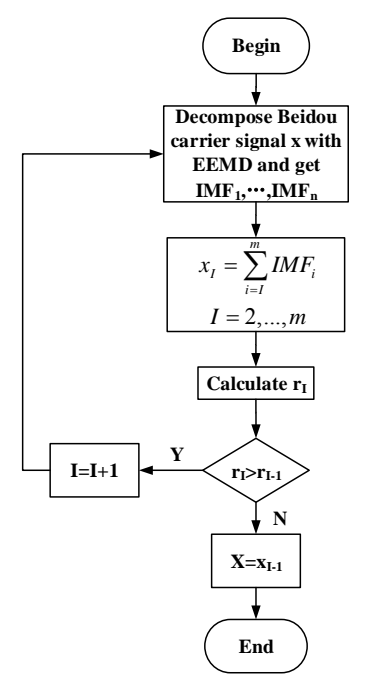

Fig.2 AIO-EEMD noise reduction flowchart

i. To get the $\mathrm{m}$ IMF components of the original Beidou carrier signal which collected by the positioning device, EEMD method is adopted to decompose the signal. The residual $r$ in formula (5) is denoted as $\mathrm{IMF}_{\mathrm{m}}$ as the last component.

ii. The high frequency component from the signal will be removed in order from $\mathrm{IMF}_{1}$ to $\mathrm{IMF}_{\mathrm{I}}$, and then combining the remaining $\mathrm{m}-1$ components together as $\mathrm{x}_{\mathrm{I}}$. iii. The index $r_{I}$ from the reconstructed signal $\mathrm{x}_{\mathrm{I}}$ that removing the component from $\mathrm{IMF}_{1}$ to $\mathrm{IMF}_{\mathrm{I}}$ will be calculated, and comparing the two adjacent indexes $r_{I}$ and $r_{I-1}$. If $r_{I}$ is greater than $r_{I-1}$, it means that the performance from the signal reconstructed by $I$ to $m$ is better than that of $I-1$ to $m$. So the signal can be further improved by removing the next order IMF component, and then let I increase by 1 continues to reconstruct the signal until the value $r_{I}$ is smaller than that of $r_{I-1}$. Then the reconstructed signal at this time $\mathrm{X}=\mathrm{x}_{\mathrm{I}-1}$ is the final denoising result. That is to say, the reconstructed result from the Beidou carrier signal with EEMD method has reached the optical performance and the algorithm can be stopped.

\section{The denoising experiment for Beidou carrier signals based on AIO-EEMD algorithm}

\subsection{Reconstruction experiment with different IMF components}

In our experiment, it's supposed that the intensity of Gaussian white noise is 0.28 . In addition, since the frequency of Beidou carrier signal is very high, the execution time and total number of IMF component have been set to 1 second and 11 respectively. The AIO-EEMD noise reduction experiment has been performed on the Beidou carrier signal which collected by a positioning device. The model of the device is ZX302, which is showed in Fig.3.

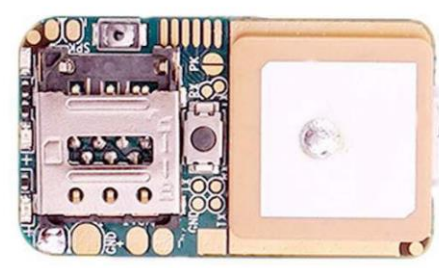


Fig.3 The ZX302 locator device

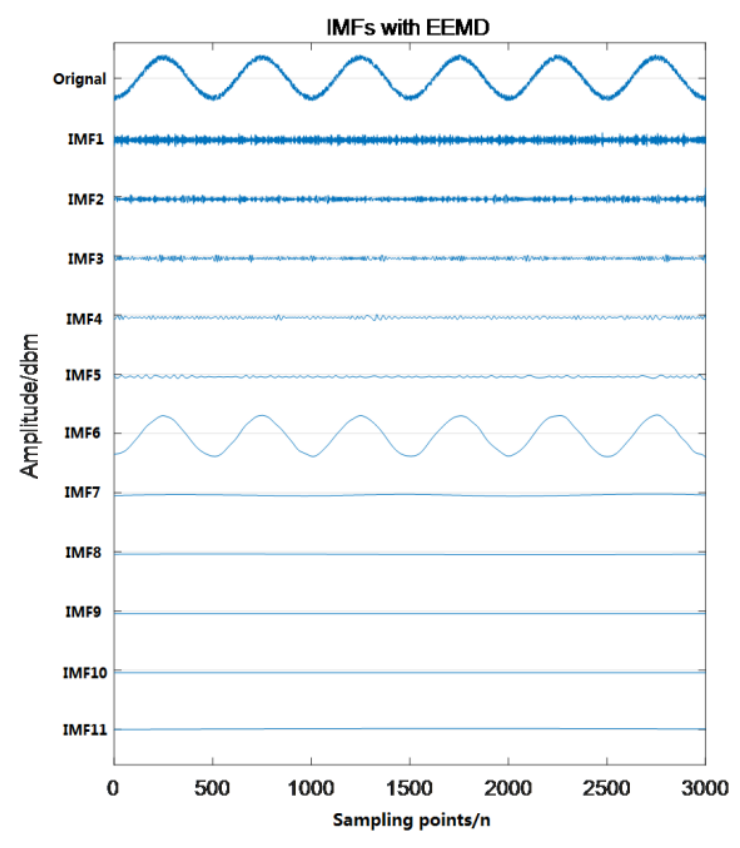

(a)
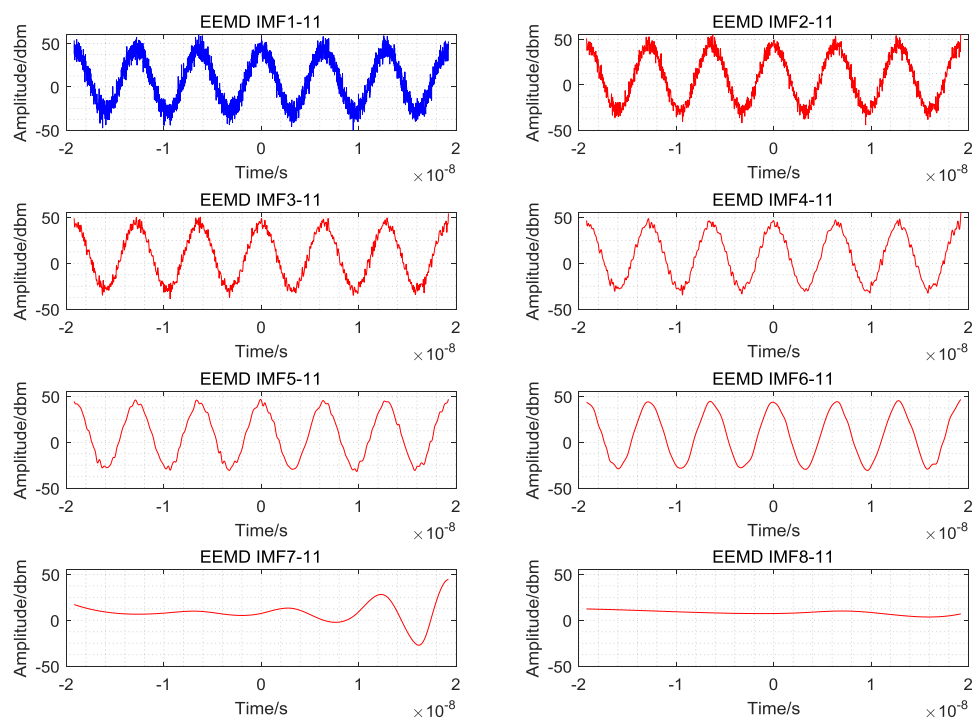

(b)

Fig.4 Experiment Results of the signal sample

(a) IMF components of the Beidou satellite carrier signal

(b) Comparison of reconstruction results of different IMF components

The original signal and IMF components from $\mathrm{IMF}_{1}$ to $\mathrm{IMF}_{11}$ have been shown in Fig.4(a). It can be seen clearly there are 11 IMF components of the orignal signal. $\mathrm{IMF}_{1} \sim \mathrm{IMF}_{5}$ are the low-order noises. Then an experiment to reconstruct the original signal with the different IMF components above has 
been carried out and the results have been shown in Fig.4(b). It can be seen the first six reconstructed signal has become more and more smooth, which means its noise is less than before. Then the reconstructed signal with $\mathrm{IMF}_{7} \sim \mathrm{IMF}_{11}$ and $\mathrm{IMF}_{8} \sim \mathrm{IMF}_{11}$ both have been distorted, in which the modal aliasing is apparent. Thus, we can deduced that the signal reconstructed with $\mathrm{IMF}_{6} \sim \mathrm{IMF}_{11}$ is the smoothest among the eight reconstructed signals. Further, the four indicators of the reconstructed signals from $\mathrm{IMF}_{1} \sim \mathrm{IMF}_{11}$ to $\mathrm{IMF}_{8} \sim \mathrm{IMF}_{11}$ are shown in Table 1.

Table 1 Denoising indicators of the Beidou Carrier Signal sample

\begin{tabular}{|c|c|c|c|c|}
\hline $\begin{array}{c}\text { IMFs for } \\
\text { Reconstruction }\end{array}$ & IMF1-11 & IMF2-11 & IMF3-11 & IMF4-11 \\
\hline SNR & 33.4843 & 34.0462 & 34.8167 & 35.1765 \\
\hline SE & 5.1195 & 4.8855 & 4.6677 & 4.5784 \\
\hline $\mathrm{R}$ & 0.9808 & 0.9820 & 0.9835 & 0.9841 \\
\hline $\mathrm{r}$ & 34.6604 & 35.2329 & 36.0145 & 36.3729 \\
\hline IMFs for & IMF5-11 & IMF6-11 & IMF7-11 & IMF8-11 \\
\hline SNR & 35.6420 & 35.8075 & 24.5722 & 20.1646 \\
\hline SE & 4.4571 & 4.4100 & 25.8493 & 26.8219 \\
\hline $\mathrm{R}$ & 0.9849 & 0.9852 & 0.0916 & 0.0898 \\
\hline $\mathrm{r}$ & 36.8512 & 37.0149 & 24.6251 & 20.2916 \\
\hline
\end{tabular}

By analyzing the data in Table 1, the variation trend of the four indicators of the reconstructed component obtained from $\mathrm{IMF}_{1} \sim \mathrm{IMF}_{11}$ to $\mathrm{IMF}_{6} \sim \mathrm{IMF}_{11}$ is increasing. When the signal is reconstructed by $\mathrm{IMF}_{7} \sim \mathrm{IMF}_{11}$, all the four indicators are decreased. That is to say, the four indicators SNR, 1/SE, $\mathrm{R}$ and $r$ from the signal reconstructed by $\mathrm{IMF}_{6} \sim \mathrm{IMF}_{11}$ are all reached their maximum value. The change trend of the index is almost the same as that of the curve. Thus, we can further infer that the noise reduction performance from $\mathrm{IMF}_{6} \sim \mathrm{IMF}_{11}$ in the proposed algorithm is the best.

\subsection{Denoising experiments with different algorithm}

In order to verify the effect of the proposed algorithm, the EMD low-pass filtering, $\mathrm{db} 3$ wavelet decomposition, ordinary EEMD and AIO-EEMD algorithms have been used to reduce the signal noise mention before respectively. The detailed result of the experiment are shown in Fig.5. 

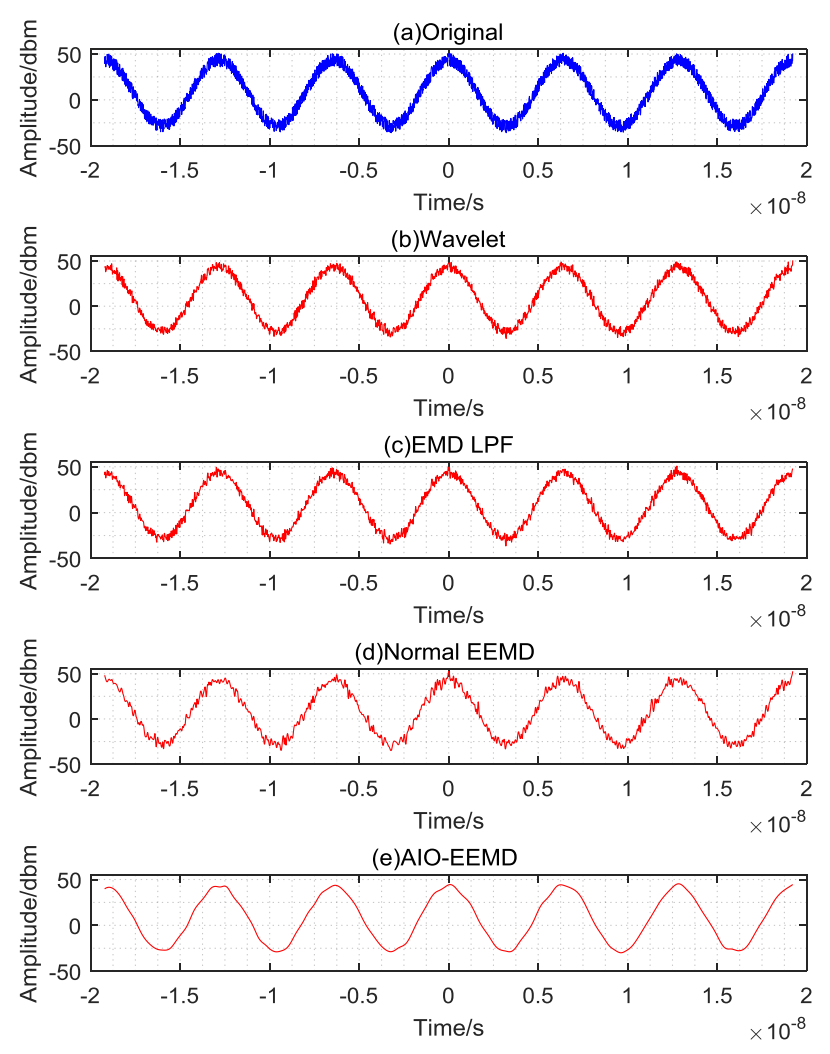

Fig.5 Comparison of noise reduction effect between AIO-EEMD and other algorithms
(a) Original carrier signal with noise,
(b) Results of EMD low-pass filter,

(c) Results of Wavelet decomposition,

(d) Results of ordinary EEMD,

\section{(e) Results of AIO-EEMD}

In the above figures, the curves obtained by the first three methods are rough, and the curves obtained by the AIO-EEMD method are relatively smooth. Therefore, we can preliminarily infer that the noise reduction performance of AIO-EEMD method is superior to the other three. In addition, we also calculate the noise reduction indexes for the above four methods respectively. The results are shown in Table 2.

Table 2 Noise reduction indexes of the four algorithms

\begin{tabular}{|c|c|c|c|c|}
\hline & $\boldsymbol{S N R}^{\prime} / \mathbf{d B}$ & $\boldsymbol{S E}^{\prime}$ & $\boldsymbol{R}^{\prime}$ & $\boldsymbol{r}^{\prime}$ \\
\hline Wavelet & 30.0865 & 5.8753 & 0.9524 & 31.2091 \\
\hline EMD LPF & 31.2765 & 5.9002 & 0.9775 & 32.4234 \\
\hline EEMD & 33.4843 & 5.1195 & 0.9808 & 34.6604 \\
\hline AIO-EEMD & 35.8075 & 4.4100 & 0.9852 & 37.0149 \\
\hline
\end{tabular}


The values of the four indicators SNR, SE, R and $\mathrm{r}$ from the AIO-EEMD method are 35.8075, $4.4100,0.9852$, and 37.0149 respectively. By comparing the above four sets of data, it can be seen that all the four indicators from the AIO-EEMD algorithm are the largest in their corresponding set. It is also consistent with the intuitive effect observed from the noise reduction curves. So, we can concluded that the AIO-EEMD algorithm has a better noise reduction performance on Beidou carrier signal than the other three algorithms.

Combining the results from the above experiments, it can be concluded that the algorithm proposed in our paper is effective.

\section{Conclusion}

Based on the characteristics of Beidou carrier signal and the steps of the basic EEMD algorithm, an adaptive index optimization EEMD (AIO-EEMD) algorithm for the noise reduction of Beidou carrier signals has been proposed in this paper. The algorithm uses an optimization index for the number of signal decomposition and reconstruction component which is consists of SNR, standard deviation SE and correlation coefficient R. In order to verify the reliability of the proposed algorithm, the noise reduction experiment on the carrier signal collected by the locator has been carried out. In addition, the denoising performance with the EMD low-pass filtering, $\mathrm{db} 3$ wavelet decomposition and basic EEMD algorithms has been compared with. The experiment results indicate that the proposed method show better performance than the other three methods in noise reduction. Thus, it can be concluded that the algorithm proposed in this paper is reliable and effective for the denoising of Beidou carrier signals.

\section{Acknowledgement \& Funding}

The research work of this paper is supported by University Synergy Innovation Program of Anhui Province (GXXT-2019-048) and National Natural Science Foundation of China "Research on Accuracy Improvement Method of Beidou Space Signal Based on Optical Fiber Time-Frequency Transmission" (41904034).

\section{Data availability statement}

The data that support the findings of this study are available from the corresponding author upon reasonable request [20].

\section{Competing interests}

No conflict of interest exits in the submission of this manuscript, and manuscript is approved by all authors for publication. I would like to declare on behalf of my co-authors that the work described is original research that has not been published anywhere previously, and not under consideration for publication elsewhere, in whole or in part. All the authors listed have approved the manuscript that is enclosed.

\section{Authors' contributions}

Wei Li, Kai Zhang and Gang Lv are responsible for the design of the proposed algorithm, the programming, data acquisition, noise reduction simulation, etc., and have jointly participated in the writing and modification of the paper. Guibao Xu and Anyi Xu are responsible for providing the Beidou Positioning device and its relevant positioning data and other experimental conditions support. 


\section{References}

[1] Beidou satellite navigation system development report (version 3.0) [R]. China Satellite Navigation System Management Office, 2018.

[2] Collin F, Warnant R. Manuscripta Geodaetica: Application of the wavelet transform for GPS cycle slip correction and comparison with Kalman filter[J]. 1995

[3] N. E. Huang, Z. Shen, S. R. Long, M. C. Wu, E. H. Shih, Q. Zheng, C. C. Tung and H. H. Liu, "The empirical mode decomposition method and the Hilbert spectrum for non-stationary time series analysis," Proceedings of the Royal Society of London, 454A, 1998, pp. 903-995.

[4] Fang Jun-long, Yu Xiao-juan, Wang Rui-fa, Wang Run-tao, Li Peng-fei, Shao Chang-hui. Research on Anti-Noise Processing Method of Production Signal Based on Ensemble Empirical Mode Decomposition(EEMD)[J]. Journal of Northeast Agricultural University (English Edition), 2017, 24, (04), pp. 69-79.

[5] Chun-sheng Wang, Chun-yang Sha, Mei Su, Yu-kun $\mathrm{Hu}$. An algorithm to remove noise from locomotive bearing vibration signal based on self-adaptive EEMD filter[J]. Journal of Central South University, 2017, 24(2), pp. 478-488.

[6] Shuhao Chen, Xuchu Mao. Research and Implementation of Beidou-3 Satellite Multi-Band Signal Acquisition and Tracking Method[J]. Journal of Shanghai Jiaotong University(Science),2019, 24(05):571-578.

[7] Luo Muzhen. Research and implementation of Beidou signal acquisition method based on FPGA[D]. Hunan University, 2017.

[8] Beidou satellite navigation system spatial signal interface control file public service signal (version 3.0) [R]. China Satellite Navigation System Management Office, 2019.
[9] Beidou Satellite Navigation System Open Service Performance Specification (Version 2.0) [R]. China Satellite Navigation System Management Office, 2018.

[10] LIU Mengmeng, WANG Min, XIONG Hui et al. In-band noise filtering of ECG signals based on EMD method[J]. Journal of Tianjin Polytechnic University, 2014, 33(4): 62-66.

[11] Yang Tao, Le Youxi, Zeng Xiande, Cai Junxiong, Zeng Yi,. Research on CEEMD Global Threshold Denoising Method Based on Autocorrelation Function[J]. Progress in Geophysics, 2018, (4): 53-57.

[12] Wang Wei, Li Zhenchun, Wang Deying. Research on wavelet threshold denoising method for seismic data based on CEEMD[J]. Geophysical Prospecting for Petroleum, 2014, 53(2): 164-172.

[13] Zhao Xiaoyu. Adaptive spectral preprocessing method based on EMD and EEMD and its application [D]. Yanshan University, 2015.

[14] Jian Zhang,Ruqiang Yan,Robert X. Gao,Zhihua Feng. Performance enhancement of ensemble empirical mode decomposition[J]. Mechanical Systems and Signal Processing, 2010, 24(7):2104-2123.

[15] Cao Ying, Duan Yubo, Liu Jicheng. Modal aliasing in Hilbert-Huang transform[J]. Vibration. Testing and Diagnosis, 2016, 36(03): 518-523+605-606.

[16] Zhang Ting, Li Shuangtian. Research on Improved EEMD Algorithm for Time Domain Aviation Electromagnetic Signal Denoising[J]. Signal Processing, 2016, 32(07): 771-778.

[17] Kai Zhang, Yujun Zhang, etc. Study on the Noise Reduction of Vehicle exhaust $\mathrm{NO}_{\mathrm{X}}$ Spectra based on Adaptive EEMD Algorithm[J]. Journal of Spectroscopy, Vol.2017, Article ID 3290420, 2017. 
WSEAS TRANSACTIONS ON SIGNAL PROCESSING DOI: 10.37394/232014.2020.16.17

[18] S. Ghofrani, H. Akbari. Comparing nonlinear features extracted in EEMD for discriminating focal and non-focal EEG signals[P]. International Conference on Signal Processing Systems, 2019.

[19] Li Hua, Liu Tao, Wu Xing, Chen Qing. Application of EEMD and improved frequency band entropy in bearing fault feature extraction $[\mathrm{J}]$. ISA transactions, 2018.

[20] Kai Zhang (2020), "Research on the Denoising of the Beidou Carrier Signal based on EEMD Algorithm with Adaptive Reconstruction Optimizing of IMF Numbers", Name of repository, Dataset, https://pan.baidu.com/s/1q

8K1b0a8Qqw_bus-5rT0Yw. Send an email to ask for the download password.
Wei Li, Kai Zhang, Gang Lv,

Guibao Xu, Anyi Xu

Creative Commons Attribution License 4.0 (Attribution 4.0 International, CC BY 4.0)

This article is published under the terms of the Creative Commons Attribution License 4.0

https://creativecommons.org/licenses/by/4.0/deed.en US 